\title{
Constraints of Developing Natural Gas Combined Cooling, Heating and Power (CCHP) Systems
}

\author{
Ke Li \\ Department of Power Engineering, School of Energy, Power and Mechanical Engineering, North \\ China Electric Power University, Baoding, Hebei 071003, PR China;
}

15933932038@163.com

Keywords: Natural Gas, CCHP System, Constrains.

\begin{abstract}
The Chinese government has put forward specific demands for the development of natural gas distributed energy, but there are still a number of constraints in the development process. This paper describes the technical principles of natural gas CCHP systems, analyzes its positive role, and demonstrates the main factors that restrict the CCHP market of the Chinese government exhaustively, combined with the current development of domestic and foreign natural gas CCHP systems.
\end{abstract}

\section{Introduction}

Natural gas distributed energy is a modern way of energy supply based on clean natural gas as fuel, it can achieve cascade utilization of energy through CCHP manner , therefore the comprehensive energy utilization efficiency can reach more than $70 \%$, and it can achieve energy supply nearby. It is an important way of efficient use of natural gas. Natural gas distributed energy is more than twice the power generation efficiency of conventional thermal power. With the acceleration of China's industrialization and urbanization process, municipalities, and regions are planning to construct new industrial development zone in suburban, and to construct commercial development zone in the city center. The common feature of this new district is in addition to a lot of power consumption, there is also a huge demand for industrial steam and some energy demand like heat and cold energy used for daily life. Given the recent reflecting from all over, practically, Chinese natural gas distributed energy however does not flourish. .In this regard, I believe that there are still some constraints of China's current development of natural gas distributed energy. Chinese government must take comprehensive measures to solve these problems as soon as possible to promote the development of the industry.

\section{Chinese and Foreign CCHP Development Situation}

\subsection{China CCHP Development Situation.}

In China, CCHP market is just in its infancy; few existing projects have been completed and put into operation. A few energy service companies initially have the ability to independently complete CCHP system planning, and the ability to design, construct, commission and operations management. However, CCHP system projects in all regions are faced with the resistance, mainly affected by some factors including incomplete supporting policies, unreasonable benefit pattern, inadequate technical support, and reliance of core devices on imports. There are a number of projects running mainly in Beijing, Shanghai and other medium-sized cities of China. With the popularity of low-carbon development in the field of urban planning and construction of China, Chinese government administrations have begun to attach importance to the application of CCHP technology and formulated relevant policies. Shanghai has recently promulgated a document named Distributed Energy Systems Engineering Technical Specification, which is the first regional technical specification that proposed detailed building requirements for the CCHP system.

\subsection{Foreign CCHP Development Situation.}

Internationally, the natural gas consumption in developed countries accounted for most proportion of energy consumption. therefore, from the 1970s, CCHP technology has been promoted and applied 
in some developed countries, but the rapid development in the true sense is during the last ten years.A few developed countries have formed a standardized manufacturing system, technology and management system in the field of CCHP.At the same time, government authorities are strongly encouraging to implement CCHP projects, and is ready to promote the CCHP system which is regarded as the main form of distributed energy. Now CCHP system has been fully entered a mature and stable market operation stage. For example, the United States installed CCHP system in many universities and office buildings. It proposed Distributed Energy Creative and the Program of Distributed Energy in 2020 in 1999, and clearly put forward that there would be 50\% new commercial facilities and university facilities using the CCHP system, and 15\% built commercial facilities and university facilities using the CCHP system by 2020; In Europe, many European countries develop CCHP systems and other energy-efficient technologies preferentially once again, due to the energy and environment pressure as well as the effect of Kyoto Protocol, and a lot of CCHP system has already been put into operation in Europe. Recent generating capacity of foreign CCHP system statistics is shown in Table 1.

Table 1 Recent generating capacity of foreign CCHP system statistics

\begin{tabular}{cccc}
\hline Nation & $\begin{array}{c}\text { Total generating capacity } \\
\text { (TW.h) }\end{array}$ & $\begin{array}{c}\text { Generating capacity of CCHP } \\
\text { (TW.h) }\end{array}$ & Proportion \\
\hline USA & 3970.6 & 162.2 & 4.1 \\
UK & 393.0 & 28.1 & 7.2 \\
Germany & 609.0 & 125.0 & 20.5 \\
Japan & 1140.0 & 190.6 & 16.7 \\
Canada & 588.0 & 65.0 & 11.4 \\
\hline
\end{tabular}

\section{Development Constraints in China}

\subsection{The System Cost Greatly Affected by Government Intervention}

Fuel accounts for $67 \% \sim 78 \%$ CCHP costs, its economic benefits influenced by market fuel and electricity prices is related to the government's determination of the price, especially in China. The gas supply price from gas company is higher than gas-fired plants, increasing the cost of power generation.The utilization of the gas seasonal spreads and other preferential system is very important .CCHP promotion requires some preferential policies, so that investors would love to offer concessional loans access and preferential tax.Otherwise it may lead to increase in energy efficiency and financial deficit while investors claim short payback period .

\subsection{Stringent Requirements for Thermal Load}

The prime condition to use CCHP is not only existing a large heating load but also a stable cooling and heating load. Although the power generation efficiency of the Micro-turbines has been increased from $17 \%-20 \%$ to $26 \%-30 \%$ until now, thermal power conversion efficiency of simple distributed power supply system whose power is from the microturbine is still much smaller than the large centralized power plant. If CCHP is only used for generating, regardless of the remaining value of the waste heat, it will cause the cost of electricity is higher than the current average price of electricity, generating alone is not economical. If a building has the large thermal load or low load variation rate, its energy utilization efficiency is generally difficult to achieve the desired effect, and the life of the generator will be affected.

\subsection{Market Factors}

Our CCHP market is short of professional system integration companies or energy service companies. It is difficult for those few existing companies to meet the development requirements in healthy competition.

The type of equipment production and supply is insufficient. Additionally, technological backwardness, poor environment adaptability, disappointing product quality, and the key technologies which is heavily dependent on imports are very serious limitations. 


\subsection{Evaluation Indexes}

The expectations from Chinese government and customers for CCHP system are consistent, but what they focus on is different. Government was concerned about efficiency, environmental protection and social benefits; however customers are more concerned about the costs, safety and convenience. For this reason, the need for a reasonable balance point for both sides is an emergency. The difficulty to make it real is a severe limitation for the coordination with CCHP systems and other technologies, which has objectively damaged the investment enthusiasm of the customers'.

\subsection{The Practical Effect and Promotion}

Currently in China, operational CCHP systems are seldom. The amount of representative CCHP projects is less if one evaluate it by the conditions of use and technology types .Furthermore, the scientific summary of experience for a comprehensive test of operation effect and subsequent project evaluation is inadequate, which affects the proposal of related policies and standards about CCHP .It also restricts the sustained promotion and favorable development of CCHP systems in China.

The statistical proportion of recent national natural gas in primary energy consumption is shown in Table2.

Table 2 The Statistical Proportion of Recent National Natural Gas in Primary Energy Consumption

\begin{tabular}{ccc}
\hline Nation & $\begin{array}{c}\text { Natural gas consumption } \\
\left(\text { billion } \mathrm{m}^{3} \text { ) }\right.\end{array}$ & $\begin{array}{c}\text { Proportion of primary energy } \\
\text { consumption }(\%)\end{array}$ \\
\hline USA & 657.2 & 26.1 \\
Canada & 100.0 & 27.3 \\
Russia & 420.2 & 55.3 \\
Japan & 93.7 & 16.6 \\
India & 41.4 & 8.6 \\
China & 80.7 & 3.6 \\
\hline
\end{tabular}

\subsection{Technical Factors}

\subsubsection{Load Forecasting}

In China, there are two general guiding concepts of the CCHP system design which are "ordering power by heat" and ordering heat by power" respectively. However, the two guiding concepts are both one-sided.The undesirable benefit of partial CCHP systems is essentially because of the incorrect predict for the heat load and electrical load. After the crew assembly is completed, in the course of operations, the not guaranteed basic load and the extravagant unit scale will lead to wasteful investment, and the CCHP system will lose the price advantage. For example in Shanghai Municipality, the CCHP system of Huangpu District Central Hospital is in poor performance because of the unit is too large. The fuel price increase will cause a great deficit and unit outage accordingly.

\subsubsection{Scheme Optimization}

It is dominate and critical for the CCHP project's success to select process ,to configure scale and to control operation. However in present domestic project, conceptual design and verification are mainly qualitative. In particular, changes in various factors lack appropriate standards and systematic ability to control, which makes the project lack of personalized features and innovation, reducing the adaptability of the system. Therefore, in the cold heating and power supply consulting industry, it is urgent to develop a practical program optimization tool.

\subsubsection{Automation}

The CCHP system requires a complete automatic control system, which allows the CCHP system to balance the supply and demand in the range of allowable range, automatically select the working conditions, regulate the operation of the equipment and form the optimal plan.However, the level of automation system for the existing domestic triple is not above average, it is mainly because of the weak system function and persistent development lag.

\section{Summary}

The development of the CCHP system is an important form to practice the idea of low carbon development. It is an effective measure to realize energy saving and emission reduction. By the 
dissertation and analysis in this paper, we can clearly recognize that in China the development of CCHP system is still difficult as a result of the existing conditions and technical level. Meanwhile, the practice also shows that existing CCHP system is in a initial situation whit deficiency. We should better construct CCHP system with one form in which there are pilots or demonstrations, learn from the experience, and be fully prepared for the future large-scale popularization.

\section{References}

[1]. Kong X Q, Wang R Z, Huang X H. Energy optimization model for a CCHP system with available gas turbines [J]. Applied Thermal Engineering, 2005, 25(2): 377-391.

[2]. Li H, Fu L, Geng K, et al. Energy utilization evaluation of CCHP systems[J]. Energy and buildings, 2006, 38(3): 253-257.

[3]. Li S, Wu J Y. Theoretical research of a silica gel-water adsorption chiller in a micro combined cooling, heating and power (CCHP) system [J]. Applied Energy, 2009, 86(6): 958-967.

[4]. Liu M, Shi Y, Fang F. A new operation strategy for CCHP systems with hybrid chillers[J]. Applied energy, 2012, 95: 164-173.

[5]. Li C Z, Shi Y M, Huang X H. Sensitivity analysis of energy demands on performance of CCHP system [J]. Energy Conversion and Management, 2008, 49(12): 3491-3497.

[6]. Maraver D, Sin A, Royo J, et al. Assessment of CCHP systems based on biomass combustion for small-scale applications through a review of the technology and analysis of energy efficiency parameters [J]. Applied Energy, 2013, 102: 1303-1313. 